\title{
Efeito da Terapia de Restrição e Indução ao Movimento em Pacientes Hemiparéticos Crônicos Pós-AVC
}

\author{
Constraint-Induced Movement Therapy in Patients After Chronic Hemiparesis Stroke \\ Jéssica Paula Magalhães ${ }^{1}$, Miriangrei Letiere ${ }^{2}$, Adriana Teresa Silva ${ }^{3}$, \\ Carolina Kosour 4 , Luciana Maria dos Reis ${ }^{5}$
}

\begin{abstract}
RESUMO
O Acidente Vascular Cerebral (AVC) geralmente causa hemiparesia, com assimetria corporal e dificuldade nos movimentos. A Terapia de Restrição e Induçáo ao movimento (TRIM) objetiva recuperar a função do membro superior parético de pacientes após AVC. Objetivo. Avaliar o efeito da TRIM na recuperação da função motora, qualidade e habilidade dos movimentos do membro superior em pacientes hemiparéticos crônicos após AVC. Método. O estudo foi realizado com cinco pacientes apresentando diagnóstico médico de AVC, com hemiparesia crônica de membro superior. Os pacientes foram avaliados pré e pós-tratamento pela escala de avaliação de Fugl-Meyer (FM) e pelo Teste de Habilidade Motora do Membro Superior (THMMS). $\mathrm{O}$ tratamento consistiu na aplicação de um protocolo modificado da TRIM, por um período de 2 semanas, 5 sessóes semanais, com duraçâo de 3 horas, totalizando 10 sessões. Resultados. No THMMS, foi observada melhora do nível de habilidade funcional e qualidade do movimento em todos os sujeitos analisados $(\mathrm{p}=0,00)$. Os resultados também evidenciaram aumento nas pontuaçóes da escala de FM $(\mathrm{p}=0,00)$. Conclusáo. Concluiu-se que a TRIM foi eficiente no tratamento desses pacientes com hemiparesia crônica secundária ao AVC, uma vez que foi observada melhora da funcionalidade do membro superior afetado após intervençấo.
\end{abstract}

Unitermos. Acidente Vascular Cerebral, Paresia, Reabilitação.

Citação. Magalhães JP, Letiere M, Silva AT, Kosour C, Reis LM. Efeito da Terapia de Restrição e Indução ao Movimento em Pacientes Hemiparéticos Crônicos Pós-AVC.

\begin{abstract}
Stroke often causes hemiparesis with body asymmetry and difficulty in movement. The Constraint-Induced Movement Therapy (CIMT) aims to recover the function of the upper limb in after stroke patients. Objective. To evaluate the effect of CIMT in the recovery of the motor function, as well as of the quality and ability of the upper limb's movements in after stroke patients with chronic hemiparesis. Method. The study was conducted with five patients having medically diagnosed stroke, with chronic upper limb hemiparesis. The patients were evaluated before and after the treatment by the Fugl-Meyer rating scale (FM) and upper limb Motor Ability Test (ULMAT). The treatment consisted of applying a modified protocol of CIMT, for a period of 2 weeks, 5 sessions/day per week, lasting 3 hours each, totaling 10 sessions. Results. In the ULMAT a significant improvement in the functional quality and ability of the upper limb's movement was observed in all analyzed subjects $(\mathrm{p}=0,00)$. The results also showed an increase in scores on the FM scale $(\mathrm{p}=0,00)$. Conclusion. It was concluded that the CIMT is an effective treatment for patients with chronic hemiplegia secondary to stroke, since an improvement in the functionality of the affected upper limb has been observed after intervention.
\end{abstract}

Keywords. Stroke, Paresis, Rehabilitation.

Citation. Magalhães JP, Letiere M, Silva AT, Kosour C, Reis LM. Constraint-Induced Movement Therapy in Patients After Chronic Hemiparesis Stroke.

\footnotetext{
Trabalho realizado no Centro Universitário da Fundaçáo Educacional Guaxupé- UNIFEG, Guaxupé-MG, Brasil.

1.Fisioterapeuta, graduada pelo Curso de Fisioterapia, Centro Universitário da Fundação Educacional Guaxupé (UNIFEG), Guaxupé-MG, Brasil.

2.Fisioterapeuta, Especialista em Neuropediatria pela Universidade Estadual de Campinas (UNICAMP); docente do Curso de Fisioterapia, Centro Universitário da Fundação Educacional Guaxupé (UNIFEG), Guaxupé-MG, Brasil. 3.Fisioterapeuta, Mestre em Ciências da Saúde - Universidade Estadual de Campinas (UNICAMP); docente do curso de Fisioterapia - Universidade Federal de Afenas (UNIFAL), Alfenas-MG, Brasil.

4.Fisioterapeuta, Doutora em Ciência Cirúrgicas- Universidade Estadual de Campinas (UNICAMP); docente do curso de Fisioterapia- Universidade Federal de Alfenas (UNIFAL), Alfenas-MG, Brasil.

5.Fisioterapeuta, Doutora em Ciências Fisiológicas- Universidade Federal de Sáo Carlos (UFSCar); docente do Curso de Fisioterapia- Universidade Federal de Alfenas (UNIFAL), Alfenas-MG, Brasil.
}

\author{
Endereço para correspondência: \\ Luciana Maria dos Reis \\ Av. Jovino Fernandes Sales, 2600 \\ Bairro Santa Clara - Prédio A \\ CEP 37130-000, Alfenas-MG, Brasil \\ E-mail: reislucianamaria@gmail.com
}




\section{INTRODUÇÃO}

O Acidente Vascular Cerebral (AVC) é definido como um rápido desenvolvimento de déficit neurológico focal com mais de 24 horas de duração e/ou lesão cerebral presente em um exame de imagem ${ }^{1}$. O AVC isquêmico representa cerca de $70 \%$ a $80 \%$ dos casos, sendo caracterizado por obstrução de uma das artérias cerebrais ou de seus ramos, interrompendo o fluxo de sangue local por mais de 24 horas, enquanto o AVC hemorrágico ocorre pela ruptura de um vaso intracraniano gerando extravasamento de sangue e aumento da pressão intracraniana ${ }^{2}$.

A incidência do AVC dobra a cada década após os 55 anos sendo, entre os 65 e 74 anos aproximadamente 14,4 por mil; entre 75 e 84 anos, 24,6 por mil e acima de 85 anos cerca de 27,0 por mil $^{3}$. Transcorrido seis meses após o AVC, 65\% das pessoas não conseguem incorporar o hemicorpo afetado em suas atividades de vida diárias (AVD's) e apenas $25 \%$ retornam às suas atividades sociais ${ }^{4}$.

Após lesão do Sistema Nervoso Central (SNC), a sequela física mais comum é a hemiparesia, definida como o comprometimento do membro superior e inferior de um mesmo lado do corpo com um padrão rígido nos músculos flexores do membro superior e extensores de membro inferior ${ }^{4}$. Estas alteraçôes causam assimetria corporal e diminuição da capacidade de descarga de peso no lado afetado, dificultando a orientação e a estabilidade na realização de movimentos com o tronco e membros, bem como a execução de atividades de vida diárias como se vestir, alimentar-se, mudar de postura, andar e sentar 5 .

A diminuição da atividade da extremidade superior está relacionada às alteraçóes de movimento e tônus além do não uso aprendido, onde os pacientes hemiparéticos perdem a memória sensório-motora e passam a utilizar apenas o membro não afetado ${ }^{6}$. O não uso aprendido baseia-se na teoria de que após uma lesão encefálica o paciente, não conseguindo utilizar o membro superior afetado nas suas atividades, passaria a restringir cada vez mais seus movimentos, como uma forma de condicionamento inibitório ${ }^{7}$.

O tratamento pós-AVC consiste em intervenções medicamentosas, apoio psicológico e tratamento fisioterapêutico. A fisioterapia tem como objetivo recuperar a função motora através de cinesioterapia, eletroterapia e técnicas de treino físico intenso, como a Terapia de
Restrição e Indução ao Movimento (TRIM), uma técnica baseada em uma pesquisa inicial realizada pelo Dr. Edward Taub entre as décadas de 70 e $80^{2}$.

A TRIM se baseia no não uso aprendido visando recuperar a função do membro superior (MS) parético de pacientes pós-lesôes encefálicas, através de treinamento intensivo de atividades motoras e restrição do MS não-parético com luva ou tipóia em 90\% do dia.

O protocolo mais utilizado da TRIM é o de 6 horas de treinamento supervisionado da extremidade parética, com restrição do MS não-parético $90 \%$ do dia durante duas semanas consecutivas ${ }^{8}$. Entretanto, outros estudos tem sido realizados no intuito de observar a eficácia da TRIM na melhora da função do membro superior em pacientes hemiparéticos ${ }^{1,4,6,7,9-11}$, utilizando diferentes protocolos de tratamento, tempo de terapia e enfoques de avaliação após intervenção.

Um estudo recente evidenciou que a TRIM é um tratamento importante na melhora funcional do membro superior parético, particularmente no que se refere ao movimento de pinça entre o polegar e o indicador. Foi observada redução do tempo necessário para realização das tarefas propostas e melhora da seletividade dos movimentos executados, avaliados por registro fotográfico e uso de cronômetro para registro do tempo de execução do movimento ${ }^{6}$.

Em outro estudo, realizado com protocolo associado da TRIM com seis horas de terapia multidisciplinar, foram observados ganhos na destreza e independência funcional do membro superior parético após $\mathrm{AVC}^{7}$.

O objetivo deste estudo foi avaliar o efeito do protocolo modificado da TRIM na recuperação da função motora, qualidade e habilidade dos movimentos do membro superior em pacientes hemiparéticos crônicos pós-AVC.

\section{MÉTODO}

\section{Amostra}

O estudo foi realizado com cinco pacientes apresentando diagnóstico médico de AVC, encaminhados a Clínica Escola de Fisioterapia pertencente ao Centro Universitário da Fundação Educacional Guaxupé (UNIFEG), localizado na cidade de Guaxupé-MG. Foram selecionados pacientes na faixa etária entre 50 e 80 anos, 
com hemiparesia crônica (mais de um ano) de membro superior, com no mínimo $20^{\circ}$ de extensão passiva de punho e $10^{\circ}$ para os dedos, além de capacidade de seguir ordens verbais, escritas ou demonstrativas ${ }^{9}$. Foram excluídos do estudo pacientes com outras patologias neurológicas associadas. A permissão para o desenvolvimento da pesquisa foi adquirida mediante assinatura dos pacientes do Termo de Consentimento Livre e Esclarecido e aprovaçâo pelo Comitê de Ética em Pesquisa do UNIFEG (protocolo n ${ }^{\circ} 219$ ).

\section{Procedimento}

O tratamento consistiu na aplicação de um protocolo modificado da Terapia de Restrição e Indução ao Movimento (TRIM), por um período de 2 semanas, 5 sessóes semanais, com duração de 3 horas, totalizando 10 sessōes ${ }^{1}$. O membro superior sadio foi restringido com uma tipóia e o membro superior parético foi estimulado a realizar atividades como rosquear recipientes de diferentes tamanhos; pendurar panos no varal; escrever utilizando caneta e papel; utilizar uma colher para retirar o feijão de um recipiente; colocar sementes, bolinhas de gude e clips em um recipiente; retirar fichas de papel de um recipiente com feijão; virar peças de dominó; virar cartas do baralho; costurar no quadro de costura; abrir diferentes fechaduras além do treino de pinça fina com materiais lúdicos ${ }^{1,6}$.

Os pacientes foram avaliados por meio da escala de Fugl-Meyer (FM) ${ }^{12}$, desenvolvida e introduzida por Fugl-Meyer e colaboradores em 1975 com o objetivo de avaliar seis aspectos do paciente: amplitude de movimento e dor, função motora, sensibilidade, equilíbrio, coordenação e velocidade. Uma linha ordinal de 3 pontos é aplicada a cada item onde: $0=$ não pode ser realizado; $1=$ realizado parcialmente e $2=$ realizado completamente, totalizando 100 pontos para função motora normal, sendo 66 para membros superiores e 34 para membros inferiores $^{10,12}$. No presente estudo foi selecionada somente a seção para membros superiores da escala.

Foi utilizado também o Teste de Habilidade Motora do Membro Superior (THMMS) ${ }^{13,14}$ com o objetivo de mensurar aspectos quantitativos e qualitativos das AVD's que envolvem o membro superior em pacientes com sequelas de AVC. O teste é composto por 13 tarefas que reproduzem atividades cotidianas, avaliadas por uma escala que varia de 0 a 5 pontos nos seguintes itens: (a) habilidade funcional (capacidade de executar uma meta), onde 0 =não tem; 1 =muito pouco; $2=$ =ouco; $3=$ moderado; $4=$ quase normal e $5=$ normal, e (b) qualidade do movimento (quâo bem o movimento da tarefa foi executado) - onde 0 =não tem; 1 =muito pobre; 2 =pobre; 3 =moderado; $4=$ quase normal e $5=$ normal. $O$ tempo de desempenho de cada tarefa é cronometrado sendo 1 minuto para atividades unilaterais e 2 minutos para bilaterais ${ }^{13,14}$.

A primeira avaliação (pré-tratamento) foi realizada antes do início do tratamento e a segunda (pós-tratamento) após o último dia de intervenção ${ }^{1,11}$.

\section{Análise Estatística}

Os dados foram analisados por meio do cálculo da média e erro padrão da média em cada dimensão do THMMS e da escala de FM. Foi realizado o teste Kolmogorov-Smirnov para determinar a normalidade dos dados. Em seguida foi utilizado o teste de Wicoxon para comparação antes e após o tratamento em cada indivíduo para o THMMS. Para a escala FM foi utilizado o teste t. As análises dos dados foram executadas pelo programa SPSS versão 20.0. Valores de $\mathrm{p} \leq 0,05$ foram considerados significativos.

\section{RESULTADOS}

A amostra estudada constituiu de cinco indivíduos, nos quais foi observada melhora no item Habilidade Funcional do THMMS após intervenção. A Figura 1 mostra os valores de média e erro padrão de cada indivíduo antes e após o tratamento, sendo observado valor de $\mathrm{p}=0,00$ em todos os sujeitos avaliados.

A Figura 2 mostra os valores de média e erro padrão da média de cada indivíduo antes e após o tratamento, sendo observada melhora nos itens relacionados à Qualidade do movimento do THMMS em todos os indivíduos avaliados $(\mathrm{p}=0,00)$.

Foi observada também, redução do tempo para realizar as tarefas propostas pelo teste no pós-tratamento em relação ao pré-tratamento $(\mathrm{p}=0,00)$, com exceção do paciente 5 , no qual foi observada diminuição não significante do tempo de execução ( $\mathrm{p}=0,2)$ (Figura 3).

Em relação à escala FM, foi observada diferença 
estatística pré e pós-intervenção em todos os indivíduos avaliados $(\mathrm{p}=0,00)$ (Figura 4).

\section{DISCUSSÃO}

No presente estudo foi observada melhora na habilidade e função motora em todos os indivíduos do estudo, após intervenção com protocolo modificado da TRIM. Esses resultados condizem com os observados em um trabalho realizado com 12 pacientes divididos aleatoriamente em 2 grupos, onde o grupo 1 realizou a TRIM e o grupo 2 realizou a TRIM associado a 10 minutos de aplicação da FES (Estimulação Elétrica Funcional) no final de cada sessão, sendo observada melhora significativa da funçáo motora do membro superior, avaliada pela escala FM em ambos os grupos ${ }^{1}$. Resultados semelhantes também foram observados por outros autores ${ }^{10,15,16}$.

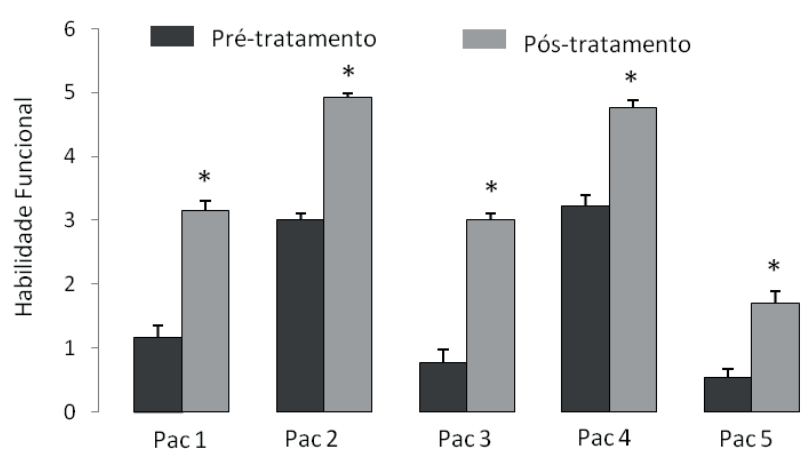

Figura 1. Registro de Habilidade Funcional avaliado por meio do Teste de Habilidade Motora do Membro Superior (THMMS) nas condições de pré e pós-tratamento. Os dados representam a média e erro padrão da média. ${ }^{*}$ p $<0,05$ em comparação ao pré-tratamento.

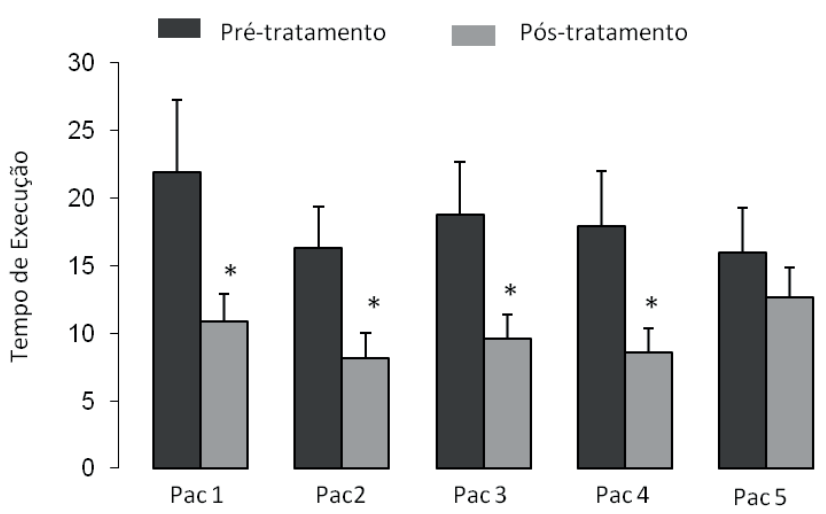

Figura 3. Registro do tempo de execução avaliado por do Teste de Habilidade Motora do Membro Superior (THMMS) nas condiçóes de pré e pós-tratamento Os dados representam a média e erro padrão da média. ${ }^{*} \mathrm{p}<0,05$ em comparação ao pré-tratamento.
Em relação ao THMMS, foi observada melhora nas três dimensóes com aumento significativo da média na habilidade funcional e qualidade do movimento, bem como redução do tempo de execução das tarefas nos cinco sujeitos avaliados neste estudo. Resultados semelhantes foram presenciados em outro estudo, onde foi aplicada a TRIM em oito pacientes avaliados pelo THMMS sendo observada melhora significativa na habilidade motora do membro superior comprometido ${ }^{11}$.

Em um estudo, realizado com 35 sujeitos com AVC crônico, divididos em três grupos aleatoriamente sendo 1) grupo TRIM (terapia com duração de meia hora, três dias por semana por dez semanas), 2) grupo reabilitação (terapia com duração de meia hora, três dias por semana por dez semanas onde $80 \%$ da terapia tinha foco na facilitação neuromuscular proprioceptiva (FNP)

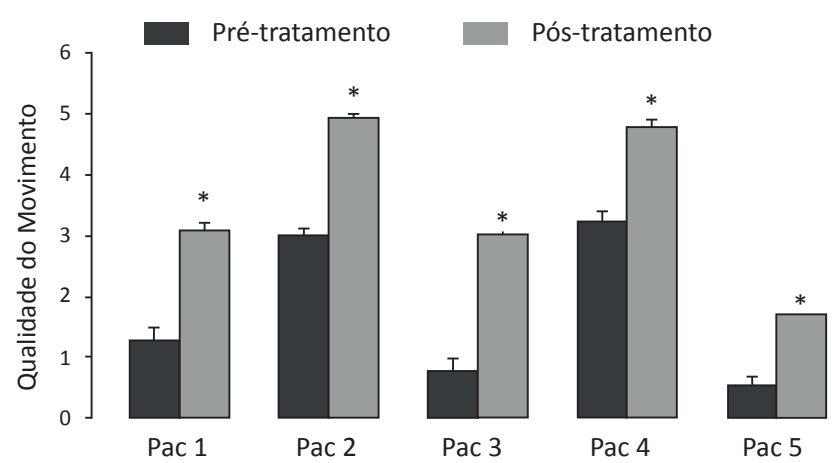

Figura 2. Registro de qualidade do movimento avaliado por meio do Teste de Habilidade Motora do Membro Superior (THMMS) nas condiçóes de pré e pós-tratamento. Os dados representam a média e erro padrão da média. ${ }^{*}$ p $<0,05$ em comparação ao pré-tratamento.

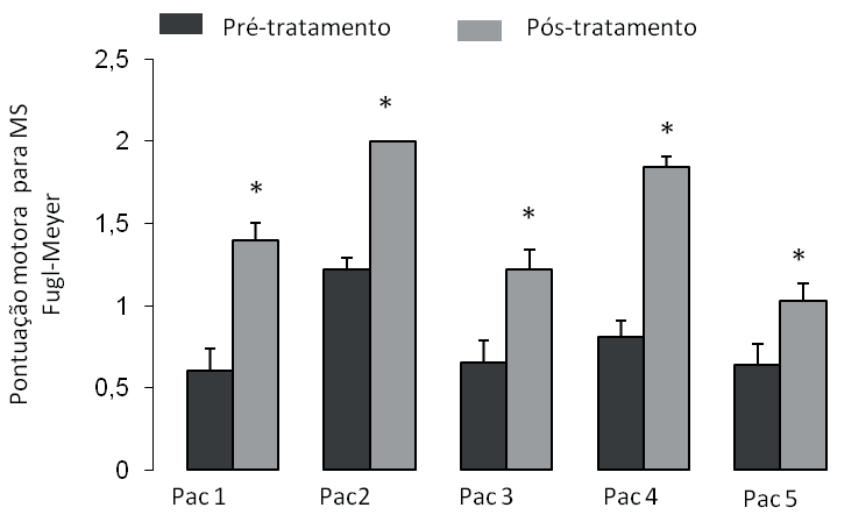

Figura 4. Pontuação obtida pela avaliação de Fugl-Meyer (FM) para membro superior nas condiçóes de pré e pós-tratamento Os dados representam a média e erro padrão da média.

* $\mathrm{p}<0,05$ em comparação ao pré-tratamento. 
e 3) grupo controle (os sujeitos não receberam tratamento durante 10 semanas), foram utilizadas as escalas Action Research Arm Test (ARAT), Fugl-Meyer (FM) e Motor Activity Log (MAL) em uma avaliação pré-intervenção e outra pós-intervenção. Os resultados demonstraram diferenças significativas na ARAT e na MAL todas a favor do grupo TRIM ${ }^{17}$.

Tem sido sugerido que a aplicação da TRIM promove reorganização cortical através do treino intensivo do membro superior comprometido pelo AVC com "superação do não uso aprendido" através da restrição do membro não afetado e consequentemente maior utilização do membro comprometido. Um estudo com estimulação transcraniana magnética focal foi realizado com o objetivo de mapear a área de saída cortical motora de um músculo da mão em ambos os lados em 13 pacientes com AVC na fase crônica da doença, antes e depois de um período de 12 dias de terapia de restrição e indução ao movimento. Foi observado que antes do tratamento, a área de representação cortical do músculo da mão afetada foi significativamente menor do que o lado contralateral. Após o tratamento, o tamanho da área de saída cortical do músculo no hemisfério afetado foi ampliada significativamente correspondendo a um melhor desempenho motor do membro parético além dos deslocamentos do centro do mapa sugerindo recrutamento de áreas cerebrais adjacentes ${ }^{18}$.

Estudos da literatura têm demonstrado a eficácia da TRIM na melhora da funcionalidade em pacientes hemiparéticos crônicos. Este efeito pode estar relacionado à melhora do tônus e do movimento voluntário, bem como da imagem corporal mediante reorganização das áreas motoras do córtex cerebral ${ }^{1,10-18}$.

Protocolos existentes na literatura demonstram que o tempo de execução da terapia não é um fator determinante para o tratamento e sim a repetição das atividades realizadas 6 . No presente estudo foi utilizado o protocolo adaptado de três horas, evidenciando melhora nas habilidades motoras assim como no protocolo original de seis horas, descrito por Taub ${ }^{2}$. Outros trabalhos utilizaram a TRIM por um período de 3 horas ${ }^{1,15,16}$, demonstrando melhora nas habilidades funcionais. Entretanto, os diferentes protocolos relacionados ao período de início de terapia e de excussão da intervenção, evidenciam a ne- cessidade de novos estudos relacionados à utilização desta técnica em pacientes após-AVC.

\section{CONCLUSÃO}

Os resultados deste estudo mostraram melhora da funcionalidade do membro superior afetado após intervenção fisioterapêutica pelo protocolo modificado da TRIM, com redução do tempo necessário para realização das tarefas propostas e aumento da qualidade e habilidade do movimento.

\section{REFERÊNCIAS}

1.Silva FPP, Gonçalves SP, Silva SB, Rios DFCR, Silva AT. Terapia de Contensão Induzida associada á eletroestimulaçáo funcional na paresia de membro superior. Rev Neurocienc 2012;20:187-93.

2.Brol AM, Bortoloto F, Magagnin NMS. Tratamento de Restrição e Indução do Movimento na reabilitaçáo funcional de pacientes pós Acidente Vascular Encefálico: uma revisão bibliográfica. Fisioter Mov 2009;22:497-509.

3.Polese JC, Tonial A, Fung FK, Mazuco R, Oliveira SG, Schuster RC. Avaliaçấo da funcionalidade de indivíduos acometidos por acidente vascular encefálico. Rev Neurocienc 2008;16:175-8.

4.Gamba RT, Cruz DMC. Efeitos da terapia de contensão induzida em longo prazo em pacientes pós- AVC. Rev Neurocienc 2011;19:735-40.

5.Cesário CMM, Penasso P, Oliveira APR. Impacto da disfunção motora na qualidade de vida em pacientes com Acidente Vascular Encefálico. Rev Neurocienc 2006;14:6-9.

6.Freitas AG, Sutani J, Pires MA, Prada SHF. Protocolo modificado da terapia de restrição em paciente hemiplégico. Rev neurocienc.2010; 18:199-203.

7.Riberto M, Monroy HM, Kaihami HN, Otsubo PPS, Battistella LR. A terapia de restrição como forma de aprimoramento da função do membro superior em pacientes com hemiplegia. Rev Acta Fisiatr 2005;12:15-9.

8.Silva LA, Tamashiro V, Assis RD. Terapia por Contensão Induzida: revisão de ensaios clínicos. Fisioter Mov 2010;23(1):153-9.

http://dx.doi.org/10.1590/S0103-51502010000100015

9.Vaz DV, Alvarenga RF, Mancini MC, Pinto TPS, Furtado SRC, Tirado MGA. Terapia de movimento induzido pela restrição na hemiplegia: um estudo de caso único. Rev Fisioter Pesq 2008;15(3):298-303.

10.Meneghetti CHZ, Silva JÁ, Guedes, CAV. Terapia de restriçấo e induçấo ao movimento no paciente com AVC: relato de caso. Rev Neurocienc 2010;18(1):18-23.

11.Bueno GDP, Lúcio AC, Oberg TD, Cacho EWA. Terapia de Restrição e Indução Modificada do Movimento em pacientes hemiparéticos crônicos: um estudo piloto. Fisioter Mov 2008;21(3):37-44.

12.Maki T, Quagliato EMAB, Cacho EWA, Paz LPS, Nascimento NH, Inoue MMEA et al. Estudo de confiabilidade da aplicação da escala de Fugl- Meyer no Brasil. Rev bras Fisioter 2006;10(2):177-83.

http://dx.doi.org/10.1590/S1413-35552006000200007

13. Cavaco NS, Alouche SR. Instrumentos de avaliação da função de membros superiores após acidente vascular encefálico: uma revisão sistemática. Fisioter Pesq 2010;17(2):178-83. 
14.Morlin ACG, Delattre AM, Cacho EWA, Oberg TD, Oliveira R. Concordância e tradução para o português do Teste de Habilidade Motora do Membro Superior- THMMS. Rev Neurocienc 2006;14(2):006-009.

15.Lee JHVD. Constraint-induced movement therapy: some thoughts about theories and eviden. J Rehabil Med 2003;41:41-5.

16.Grotta JC, Noser EA, RO T, Boake C, Levin H, Aronowski J. Constraint-induced movement therapy. Stroke 2004;35:2699-701.

http://dx.doi.org/10.1161/01.STR.0000143320.64953.c4
17.Page SJ, Levine P, Leonard A, Szaflarski JP, Kissela BM. Modified constraint- induced therapy in chronic stroke: results of a single- blinded randomized controlled trial. Phs Ther 2008;88:333-40.

http://dx.doi.org/10.2522/ptj.20060029

18.Lierpert J, Bauder H, Miltner WHR, Taub E, Weiller C. Treatment-Induced Cortical Reorganization After Stroke in Humans. Stroke 2000;31:1210-6. http://dx.doi.org/10.1161/01.STR.31.6.1210 